\title{
Combined array CGH plus SNP genome analyses in a single assay for optimized clinical testing
}

\author{
Joanna Wiszniewska ${ }^{1}$, Weimin $\mathrm{Bi}^{1}$, Chad Shaw ${ }^{1}$, Pawel Stankiewicz ${ }^{1}$, Sung-Hae L Kang ${ }^{2}$, Amber N Pursley ${ }^{1}$, \\ Seema Lalani ${ }^{1,3}$, Patricia Hixson ${ }^{1}$, Tomasz Gambin ${ }^{4}$, Chun-hui Tsai ${ }^{5,6}$, Hans-Georg Bock ${ }^{7}$, Maria Descartes ${ }^{8}$, \\ Frank J Probst ${ }^{1,3}$, Fernando Scaglia ${ }^{1}$, Arthur L Beaudet ${ }^{1,3}$, James R Lupski ${ }^{1,3}$, Christine Eng ${ }^{1,3}$, \\ Sau Wai Cheung ${ }^{1,3}$, Carlos Bacino ${ }^{1,3}$ and Ankita Patel ${ }^{\star, 1}$
}

In clinical diagnostics, both array comparative genomic hybridization (array CGH) and single nucleotide polymorphism (SNP) genotyping have proven to be powerful genomic technologies utilized for the evaluation of developmental delay, multiple congenital anomalies, and neuropsychiatric disorders. Differences in the ability to resolve genomic changes between these arrays may constitute an implementation challenge for clinicians: which platform (SNP vs array CGH) might best detect the underlying genetic cause for the disease in the patient? While only SNP arrays enable the detection of copy number neutral regions of absence of heterozygosity $(\mathrm{AOH})$, they have limited ability to detect single-exon copy number variants (CNVs) due to the distribution of SNPs across the genome. To provide comprehensive clinical testing for both CNVs and copy-neutral AOH, we enhanced our custom-designed high-resolution oligonucleotide array that has exon-targeted coverage of 1860 genes with 60000 SNP probes, referred to as Chromosomal Microarray Analysis - Comprehensive (CMA-COMP). Of the 3240 cases evaluated by this array, clinically significant CNVs were detected in 445 cases including 21 cases with exonic events. In addition, 162 cases (5.0\%) showed at least one $\mathrm{AOH}$ region $>10 \mathrm{Mb}$. We demonstrate that even though this array has a lower density of SNP probes than other commercially available SNP arrays, it reliably detected AOH events $>10 \mathrm{Mb}$ as well as exonic CNVs beyond the detection limitations of SNP genotyping. Thus, combining SNP probes and exon-targeted array CGH into one platform provides clinically useful genetic screening in an efficient manner.

European Journal of Human Genetics (2014) 22, 79-87; doi:10.1038/ejhg.2013.77; published online 22 May 2013

Keywords: array CGH; SNP; uniparental disomy; absence of heterozygosity; medically actionable variants

\section{INTRODUCTION}

Variation in the human genome consists of two major types: (1) single nucleotide variation, in the form of DNA base-pair substitutions and short indels, and (2) structural variation affecting many base pairs, including inversions, translocations, insertions, deletions, and duplications resulting in copy number variation $(\mathrm{CNV}) .{ }^{1}$ Advances in genome-wide analytical techniques, such as array comparative genomic hybridization (array $\mathrm{CGH}$ ) and single nucleotide polymorphism (SNP) genotyping, can be used to detect CNVs. The number of identified CNVs has increased dramatically as the resolution of the detection technologies has improved. ${ }^{2-7}$ Rapidly, $\mathrm{CNV}$ detection has become an integral part of genetic studies of disease susceptibility, delineation of novel genomic disorders, ${ }^{8-10}$ and analysis of data from genome-wide association studies. ${ }^{11,12}$

Both array CGH and SNP genotyping are routinely utilized by clinicians for the evaluation of patients with developmental delay (DD)/intellectual disability (ID), multiple congenital anomalies (MCA), ${ }^{13}$ and neuropsychiatric disorders. ${ }^{14}$ Genomic resolution by both array platforms (SNP and array CGH) used in clinical laboratories allows for the detection of genomic gains and losses of $\sim 400 \mathrm{~kb}$ in size ${ }^{15}$ Custom-designed oligonucleotide array CGH with greater resolution of the human genome, enabling detection of singleexon CNVs for clinically relevant genes, has also been implemented clinically. ${ }^{16,17}$ This approach increases diagnostic yield, but also increases the likelihood of detecting variants of uncertain clinical significance.

While single-exon resolution throughout the genome is not feasible for SNP arrays, SNP arrays show higher sensitivity for the detection of low-level mosaic aneuploidies and chimerism ${ }^{18}$ and offer the ability to detect copy number neutral regions of absence of heterozygosity $(\mathrm{AOH}) .{ }^{19}$ Consanguinity can be revealed by $\mathrm{AOH}$, because multiple regions of $\mathrm{AOH}$ are expected to be present in individuals from inbred populations, representing chromosomal segments that are identical by descent after transmission through parental lineages. The size and number of $\mathrm{AOH}$ blocks correlate with the degree of parental relatedness. ${ }^{20}$ Researchers and clinicians are also using the location of homozygous regions for mapping information in consanguineous families to identify autosomal recessive disease-causing genes.

${ }^{1}$ Department of Molecular and Human Genetics, Baylor College of Medicine, Houston, TX, USA; ${ }^{2}$ Allina Cytogenetics Laboratory, Abbott Northwestern Hospital, Minneapolis, MN, USA; ${ }^{3}$ Department of Pediatrics, Baylor College of Medicine, Houston, TX, USA; ${ }^{4}$ Institute of Computer Science, Warsaw University of Technology, Warsaw, Poland; ${ }^{5}$ Department of Molecular and Medical Genetics, Oregon Health and Sciences University-OHSU, Portland, OR, USA; 'Department of Pediatrics, The Children's Hospital, University of Colorado School of Medicine, Aurora, CO, USA; ${ }^{7}$ Department of Pediatrics, University of Mississippi Medical Center, Jackson, MS, USA; ${ }^{8}$ Department of Genetics, University of Alabama, Birmingham, AL, USA

*Correspondence: Dr A Patel, Department of Molecular and Human Genetics, Baylor College of Medicine, One Baylor Plaza, NAB 2015, Houston, TX 77030, USA. Tel: +1 713798 8534; Fax: +1 713798 6584; E-mail: ankitap@bcm.edu

Received 5 April 2012; revised 28 January 2013; accepted 12 March 2013; published online 22 May 2013 
When confined to a single chromosome, $\mathrm{AOH}$ regions may indicate uniparental disomy (UPD). ${ }^{21,22}$ Several mechanisms can lead to UPD, resulting in isodisomy if both transmitted homologs are identical, heterodisomy if both homologs from one parent are present or segmental isodisomy if part of the chromosome is isodisomic and the other part is heterodisomic. ${ }^{23,24}$ Although the true prevalence of UPD is not known, it is expected to be at least $\sim 1$ in 3500 live births based upon information available in the 'pre-genome analyses era. ${ }^{25}$ UPD is a well-known mechanism leading to human disease if a chromosome containing imprinted genes is involved or if a recessive disease-causing mutation is present. $^{25,26}$

We now show that, by combining SNPs with our exon-targeted oligonucleotide array, detection for both copy number variation and copy-neutral $\mathrm{AOH}$ are optimized and enabled in a convenient, single genomic assay.

\section{MATERIALS AND METHODS}

\section{Patients and sample preparation}

A total of 3240 patient samples referred to the Medical Genetics Laboratory (http://www.bcm.edu/geneticlabs/) at Baylor College of Medicine from October 2010 to March 2012 were analyzed by the Chromosomal Microarray Analysis - Comprehensive (CMA-COMP) array. As an independent experimental SNP platform and quality assurance measure, 59 consecutive cases in which an $\mathrm{AOH}$ event $(>10 \mathrm{Mb}$ ) was identified by the CMA-COMP array were also analyzed on an Illumina SNP array (Illumina Inc., San Diego, CA, USA).

All studies were performed on patient DNA extracted from peripheral blood using the Puregene DNA blood kit (Gentra, Minneapolis, MN, USA) according to the manufacturer's instructions.

\section{CMA-COMP}

Microarray design. The custom-designed 400K CMA-COMP array used in this study was manufactured by Agilent Technologies, Inc. (Santa Clara, CA, USA), and contains $\sim 280000$ oligonucleotide probes targeting 1860 genes at the exon level of genome resolution $(<500 \mathrm{bp})$, with an average backbone coverage of one probe per $30 \mathrm{~kb}$, an improved design of a previously described version by Boone et al, ${ }^{16}$ and 60000 SNP probes in duplicate.

Analysis. DNA was digested with AluI and RsaI in order to allow detection of the SNPs located at the enzymes' recognition sites. The remaining procedures of DNA labeling and hybridization were performed according to the manufacturers' instructions, with minor modifications. ${ }^{27}$

For detailed array analysis, see Supplementary I.

Reporting. CNVs were reported and classified into three categories: (1) abnormal, (2) unclear clinical significance, and (3) likely benign. Abnormal CNVs include aneuploidy, known microdeletion/duplication syndromes, genomic imbalances larger than $2 \mathrm{Mb}$, other known genomic disorders (eg, the 1.5-Mb CMT1A duplication) and copy number changes involving pathogenic single genes. In cases where a pathogenic $\mathrm{CNV}$ is identified that may be associated with a presymptomatic condition, we report these incidental findings to the referring physician in order to facilitate adequate counseling and prompt medical attention as needed, ${ }^{28}$ consistent with current ACMG recommendations. CNVs of unclear clinical significance include those smaller than $2 \mathrm{Mb}$ in size that have not been correlated with a clinical phenotype. $\mathrm{CNVs}$ are classified as likely benign if they are polymorphic in the normal population as determined by reviews of recent literature. Evaluation of potential medically actionable variants includes careful consideration of the size of the CNV, variant allele frequency, gene(s) involved, whether it represents a de novo or inherited event, and the reported phenotypic clinical findings in the child or other relevant family members. ${ }^{29,30}$

Additionally, $\mathrm{AOH}$ segments greater than $10 \mathrm{Mb}$ in size were reported.

SNP genotyping platform. SNP array analysis was performed on the Illumina Infinium HD assay platform using HumanOmnil-Quad BeadChip (Illumina
Inc.) with $200 \mathrm{ng}$ of genomic DNA according to the manufacturer's instructions. The GenomeStudio software (Illumina, Inc.) was used for data processing and analysis. $\mathrm{AOH}$ regions larger than $10 \mathrm{Mb}$ were used for comparison to the CMA-COMP results.

\section{RESULTS}

\section{Comparison of AOH calling by CMA-COMP and Illumina SNP} array

Of the 3240 samples referred for the CMA-COMP array, $162(5 \%)$ had at least one region of contiguous $\mathrm{AOH}$ larger than $10 \mathrm{Mb}$. A total of 59 consecutive cases with one or more $\mathrm{AOH}$ regions measuring $>10 \mathrm{Mb}$ in size as identified by the CMA-COMP array were also tested on the Illumina SNP array for quality control. Fifteen of these cases had interstitial, terminal, or centromeric $\mathrm{AOH}$ in single chromosomes (Table 1). The $\mathrm{AOH}$ regions were confirmed by the Illumina array in all 15 cases; however, the $\mathrm{AOH}$ calls in two cases were found to represent smaller-sized $\mathrm{AOH}$ regions by the higherresolution Illumina SNP array. Twenty seven cases had $\mathrm{AOH}$ regions totaling $100 \mathrm{Mb}$ or higher, which is suggestive of consanguinity. Such high levels of $\mathrm{AOH}$ were confirmed by the Illumina array for all these cases (Table 2). Therefore, the CMA-COMP platform, even though it has a lower density of SNP probes than the Illumina array, was able to reliably detect $\mathrm{AOH}$ events $>10 \mathrm{Mb}$.

\section{Uniparental disomy}

Two cases were identified by CMA-COMP with $\mathrm{AOH}$ in a single chromosome within which all interrogated SNPs were homozygous, indicating the presence of uniparental isodisomy. One case showed isodisomy 1 and the other isodisomy 16. Maternal UPD16 has been associated with intrauterine growth restriction and fetal malformations. ${ }^{31}$ Additionally, 45 cases analyzed by CMA-COMP showed $\mathrm{AOH}$ regions exceeding $10 \mathrm{Mb}$ involving one chromosome only, indicating possible uniparental heterodisomy for that particular chromosome. Twelve of these cases involved chromosomes that contain imprinted regions associated with a clinical phenotype [chromosomes 7 (three cases), 14 (seven cases), and 15 (two cases)]. Methylation-specific PCR was performed on two (Figures 1a and b) of the seven cases with $\mathrm{AOH}$ limited to chromosome 14; in both cases, there was no evidence of UPD. The B allele frequency plot for a case with $\mathrm{AOH}$ of $\sim 61 \mathrm{Mb}$ on chromosome 14 is shown in Figure 1c. Unfortunately, this case was lost to follow-up, so UPD could not be confirmed. For one case involving $\mathrm{AOH}$ on chromosome 15, an interstitial duplication of 15q11.2q13.1 and maternal uniparental trisomy was detected, genomic findings which are all consistent with a diagnosis of Prader-Willi syndrome. A detailed description of this clinical case is described elsewhere. ${ }^{32}$ All other remaining cases with $\mathrm{AOH}$ on imprinted chromosomes were lost to follow-up.

Of interest, one case had multiple $\mathrm{AOH}$ regions limited to chromosome 9 (Figure 2), which was confirmed by the Illumina SNP array. Analysis of the proband and parental genotypes indicated the presence of maternal heterodisomy 9 .

\section{Consanguinity}

Multiple $\mathrm{AOH}$ regions $>10 \mathrm{Mb}$ involving two or more chromosomes were identified in 115 cases by CMA-COMP, of which 44 cases were also performed on the Illumina SNP array. As shown in Table 2, the $\mathrm{AOH}$ calls between the two arrays are comparable in the 27 cases with $\mathrm{AOH}$ regions totaling $\geq 100 \mathrm{Mb}$. For cases in which the degree of parental relatedness was provided by the referring center (Table 3 ), we compared the total length of $\mathrm{AOH}$ regions to that which is expected from the coefficient of inbreeding $(F)$. Patients whose parents are first 
Table 1 Comparison of the results of CMA-COMP and Illumina array for $\mathrm{AOH}$ regions greater than $10 \mathrm{Mb}$ in size and limited to single chromosomes

\begin{tabular}{|c|c|c|c|c|c|c|}
\hline \multirow[b]{2}{*}{ Chromosome } & \multicolumn{2}{|c|}{ CMA-COMP array } & \multicolumn{2}{|c|}{ IIlumina SNP array } & \multirow[b]{2}{*}{ Location } & \multirow[b]{2}{*}{ UPD confirmed } \\
\hline & Genomic interval & Size in $M b$ & Genomic interval & Size in $M b$ & & \\
\hline 1 & $166983908-183983607$ & 17.0 & $167228031-183982100$ & 16.7 & Interstitial & - \\
\hline 3 & $38493212-52622086$ & 14.1 & $45790685-52193685$ & 6.4 & Interstitial & - \\
\hline 3 & $45708410-59299266$ & 13.6 & $47077306-53281955^{a}$ & 6.2 & Interstitial & - \\
\hline 5 & 97151 690-123037 288 & 25.9 & 106422 150-123740802 & 17.3 & Interstitial & - \\
\hline 5 & $163685643-178577218$ & 14.9 & $163735878-176745707$ & 13.0 & Interstitial & - \\
\hline 5 & $125870653-146253199$ & 20.4 & $127465300-146414993$ & 18.9 & Interstitial & - \\
\hline 7 & $107850309-120523873$ & 12.7 & $109017420-120492482$ & 11.5 & Interstitial & - \\
\hline 8 & $35551788-61354167$ & 25.8 & $37132731-63207089$ & 26.1 & Centromeric & - \\
\hline \multirow[t]{2}{*}{9} & $4175231-14383183$ & 10.2 & $1486230-14377817$ & 12.9 & Interstitial & UPD \\
\hline & $88061835-128350612$ & 40.3 & $66587429-126250362$ & 59.7 & Interstitial & - \\
\hline 12 & $1-22919015$ & 22.9 & $1607841-22154162$ & 20.5 & Terminal & - \\
\hline 14 & $35601054-50516087$ & 14.9 & $35958318-50563154$ & 14.6 & Interstitial & No UPD \\
\hline 14 & $33803584-70306504$ & 36.5 & $33742889-70200059$ & 36.4 & Interstitial & No UPD \\
\hline 14 & $32945633-94103812$ & 61.2 & $32899071-86182374$ & 53.3 & Interstitial & - \\
\hline 15 & $21317493-68226073$ & 46.9 & $26998850-68211801$ & 41.2 & Interstitial & UPD \\
\hline$x$ & $95140723-119474584$ & 24.3 & $95721890-119378541$ & 23.6 & Interstitial & - \\
\hline
\end{tabular}

asmall non-contiguous segments of $\mathrm{AOH}$ were seen within the same AOH interval identified by the CMA-COMP.

Table 2 Comparison of the results of CMA-COMP and Illumina array for detection of $\mathrm{AOH}$ regions totaling $100 \mathrm{Mb}$ or higher in size

\begin{tabular}{|c|c|c|c|c|}
\hline Patients & $\begin{array}{c}\text { AOH level } \\
(M b)\end{array}$ & $\begin{array}{c}\text { CMA-COMP array } \\
\text { Number of chromosomes } \\
\text { involved }\end{array}$ & $\begin{array}{c}\text { Number of } \\
\text { blocks }\end{array}$ & $\begin{array}{c}\text { Illumina } \\
\text { array } \\
\text { AOH level } \\
\text { (Mb) }\end{array}$ \\
\hline Case 1 & 871 & 15 & 18 & 851 \\
\hline Case 2 & 863 & 20 & 25 & 787 \\
\hline Case 3 & 707 & 16 & 23 & 702 \\
\hline Case 4 & 678 & 18 & 20 & 681 \\
\hline Case 5 & 654 & 14 & 16 & 609 \\
\hline Case 6 & 514 & 10 & 14 & 529 \\
\hline Case 7 & 501 & 10 & 14 & 506 \\
\hline Case 8 & 352 & 8 & 8 & 354 \\
\hline Case 9 & 345 & 8 & 11 & 367 \\
\hline Case 10 & 332 & 7 & 9 & 310 \\
\hline Case 11 & 289 & 9 & 10 & 247 \\
\hline Case 12 & 276 & 8 & 8 & 244 \\
\hline Case 13 & 270 & 8 & 9 & 240 \\
\hline Case 14 & 252 & 8 & 9 & 245 \\
\hline Case 15 & 234 & 5 & 6 & 147 \\
\hline Case 16 & 223 & 8 & 8 & 228 \\
\hline Case 17 & 194 & 5 & 5 & 127 \\
\hline Case 18 & 187 & 7 & 7 & 150 \\
\hline Case 19 & 177 & 6 & 7 & 158 \\
\hline Case 20 & 158 & 6 & 7 & 152 \\
\hline Case 21 & 141 & 6 & 6 & 138 \\
\hline Case 22 & 138 & 6 & 7 & 130 \\
\hline Case 23 & 129 & 5 & 7 & 117 \\
\hline Case 24 & 122 & 3 & 4 & 90 \\
\hline Case 25 & 118 & 4 & 4 & 107 \\
\hline Case 26 & 110 & 3 & 3 & 108 \\
\hline Case 27 & 107 & 5 & 5 & 96 \\
\hline
\end{tabular}

cousins (third degree of relationship) are expected to have $1 / 16$ of their genome homozygous or $\sim 179 \mathrm{Mb}$ of total $\mathrm{AOH}$ regions. The total length of $\mathrm{AOH}$ regions observed was $225 \mathrm{Mb}$ as shown in case 2. Consistent data were also observed in cases of fourth and sixth degree of parental relationship [expected $90 \mathrm{Mb}$ and observed 89 and 82 for fourth degree (cases 3 and 4), and expected $22.5 \mathrm{Mb}$ and observed 58 for sixth degree (case 5)].

Notably, our analyses identified 10 cases in which the observed total length of $\mathrm{AOH}$ regions was consistent with that expected for a first degree relationship $(F=1 / 4$ or $\sim 716 \mathrm{Mb})$. In those cases, the total length of $\mathrm{AOH}$ ranged from $\sim 506 \mathrm{Mb}$ to $851 \mathrm{Mb}$, with a mean of $677 \mathrm{Mb}$. Amongst this group of subjects, consanguinity was confirmed in two patients, one of which was known to be the product of a sister-brother mating (Table 3, case 1).

\section{Autosomal recessive disorders and $\mathrm{AOH}$}

We evaluated regions of $\mathrm{AOH}$ for gene content when the indication was a specific disease. In four patients with an autosomal recessive disorder, the associated gene localized within one of the $\mathrm{AOH}$ regions. Sequencing analysis performed elsewhere confirmed the presence of a homozygous mutation in two patients (Table 4).

One patient, the product of a consanguineous mating (second cousins once removed), presented with DD/ID and microcephaly, and MRI revealing a mild diffuse reduction in the volume of the cerebral hemispheric white matter with a borderline to increased size of the corpus callosum. CMA-COMP analysis showed $\mathrm{AOH}$ events larger than $10 \mathrm{Mb}$ on chromosomes 8 and 14. The $\mathrm{AOH}$ event on chromosome 8 also harbored a homozygous deletion of $\sim 52 \mathrm{~kb}$ involving exons 4-14 of the VPS13B (vacuolar protein sorting 13 homolog $B$ ) gene (Figures $3 \mathrm{a}$ and $\mathrm{b}$ ), which is associated with Cohen syndrome (OMIM \#216550) and consistent with the clinical phenotype of this patient.

\section{Intragenic CNVs}

An advantage of the CMA-COMP array platform is its ability to detect very small, single-exon copy number changes. Pathogenic genomic deletions and/or duplications were detected in $14 \%$ (445/3240) of the cases. CNVs of unclear clinical significance were detected in an additional 13\% (421/3240). Intragenic pathogenic CNVs were detected in 21 cases (Table 5) with an average of 21 probes (range 4-90). The smallest CNV was confirmed by sequencing to be 


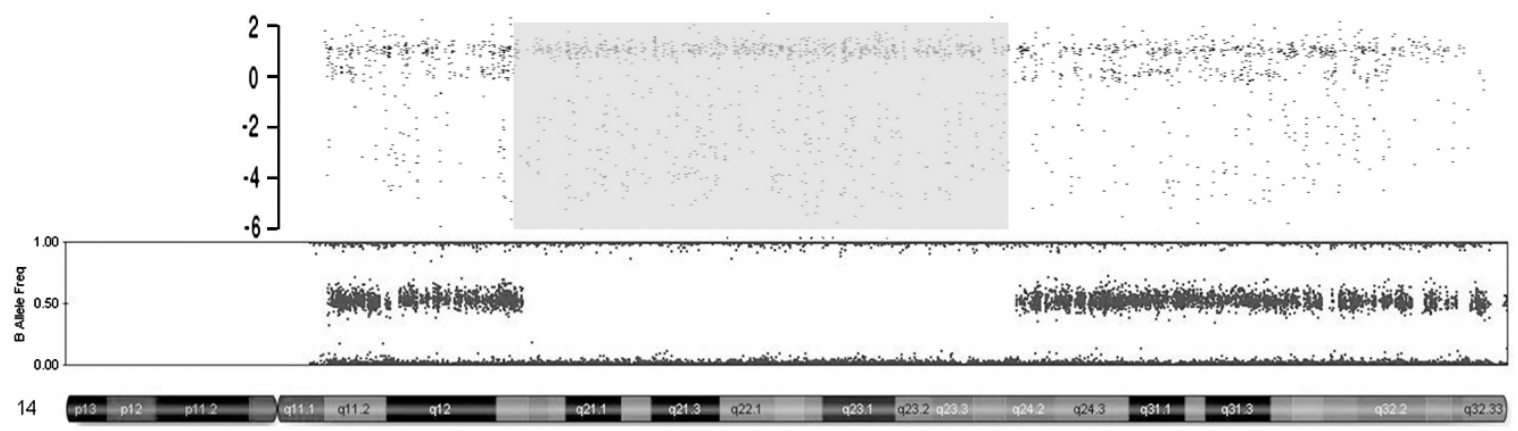

b
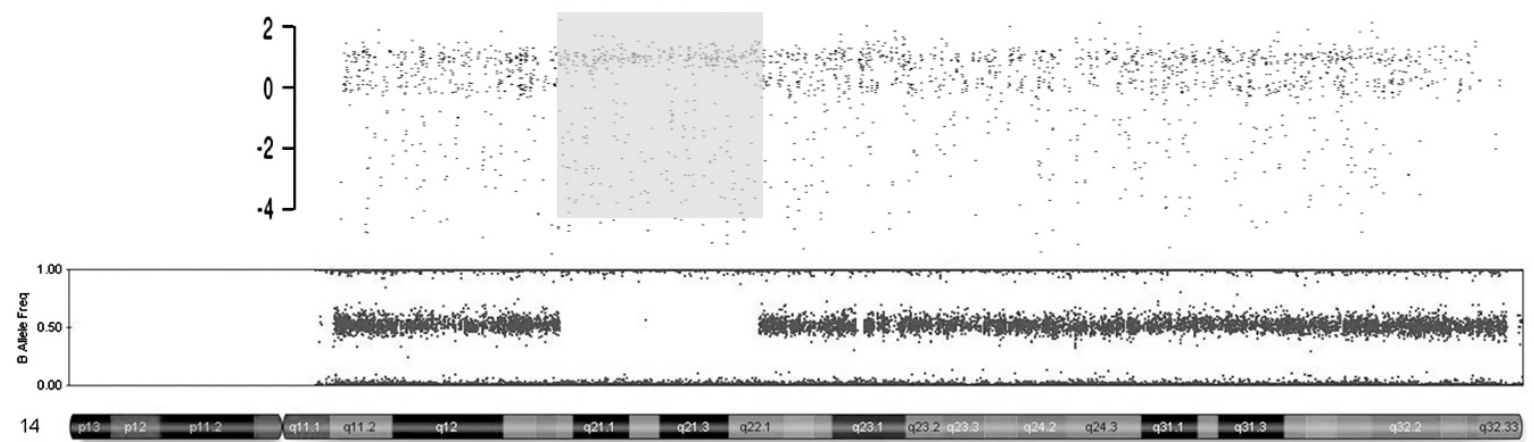

c
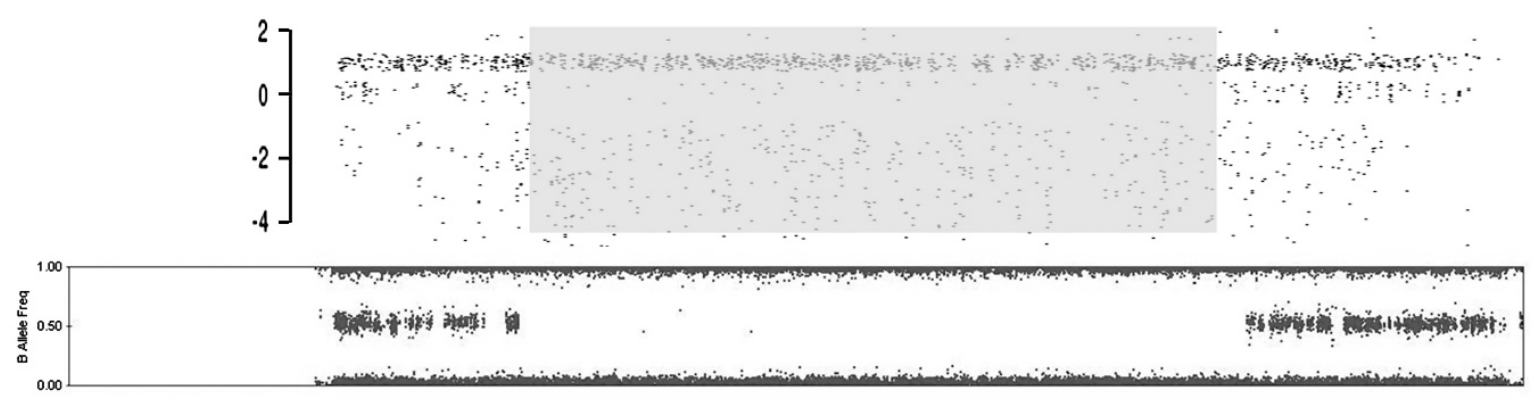

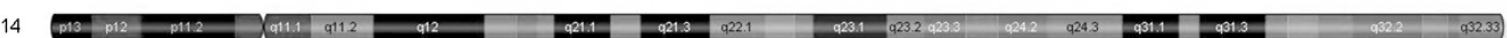

d
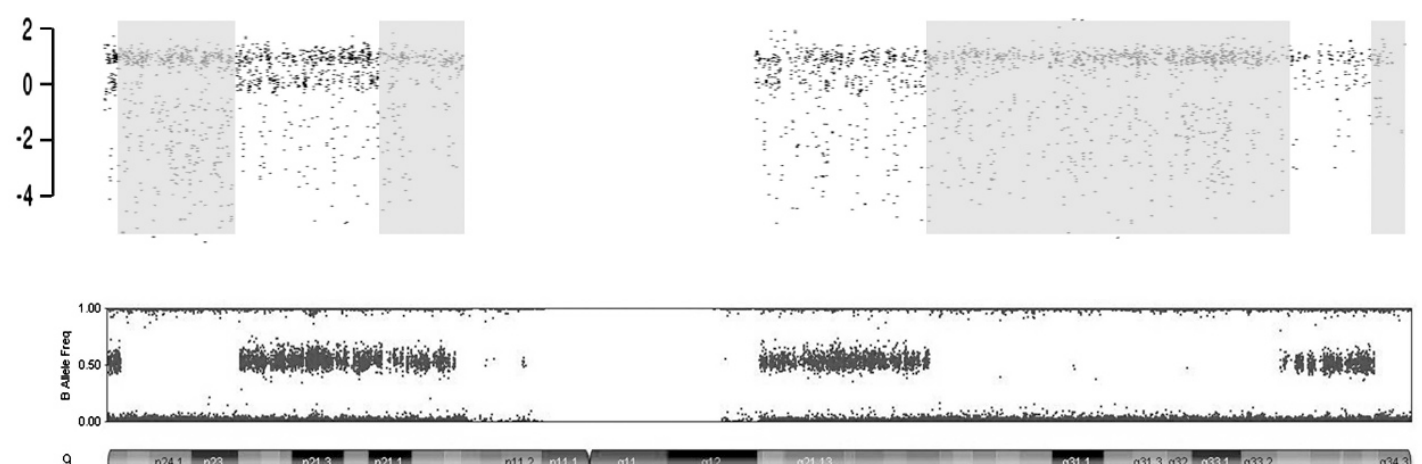

Figure 1 Large regions of $\mathrm{AOH}$ limited to single chromosomes suggestive of heterodisomy. Top panels - CMA-COMP SNP data. Bottom panels corresponding Illumina B allele frequency data. (a-c) Chromosome 14. Follow-up studies by methylation specific PCR showed presence of both maternal and paternal bands, indicating biparental chromosome 14 inheritance for cases shown in panels (a) and (b). Methylation studies were not available for the case shown in panel (c). (d) Chromosome 9. Follow-up parental SNP array analyses confirmed presence of maternal heterodisomy in this patient. 
1

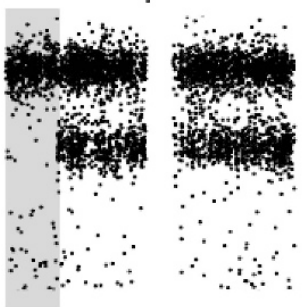

6

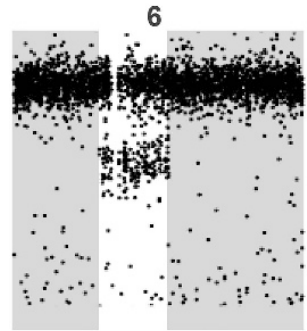

11

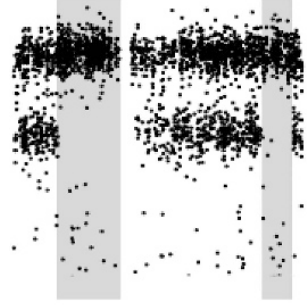

16

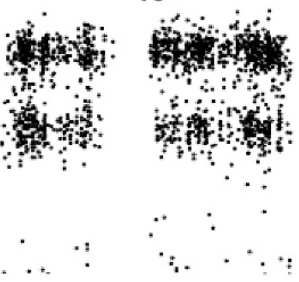

21.

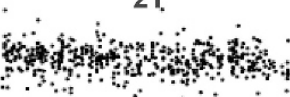

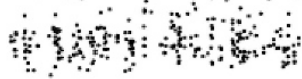

2

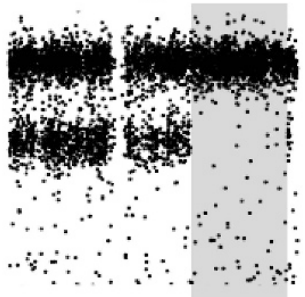

7

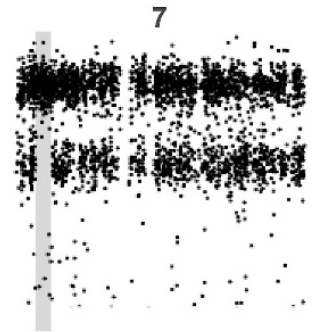

12

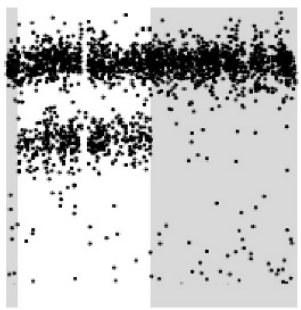

17

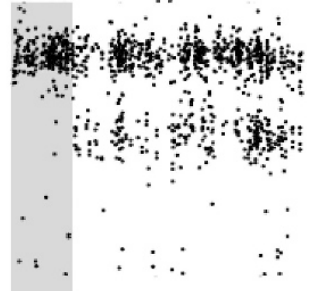

22

कासे क्ष

a d o
3

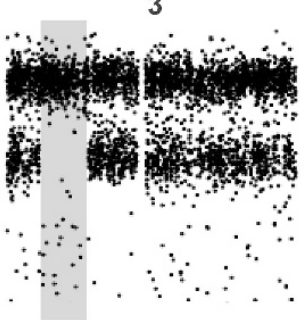

8

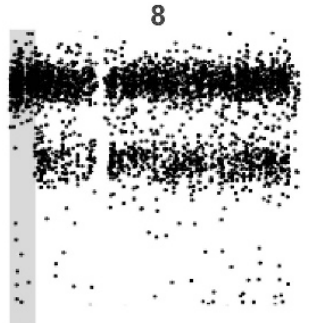

13

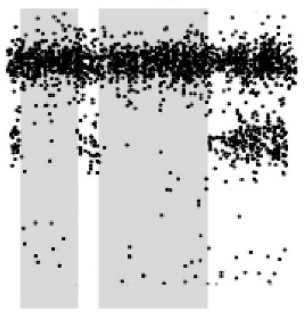

18
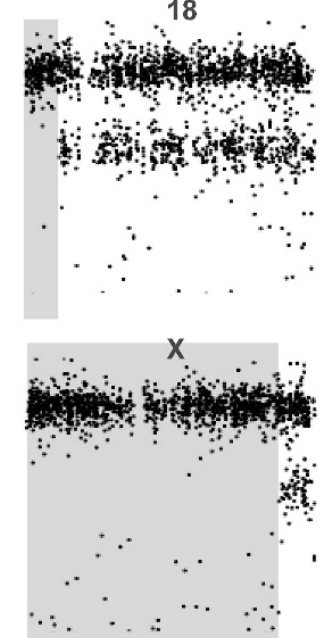

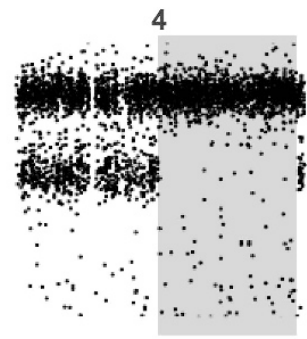

9

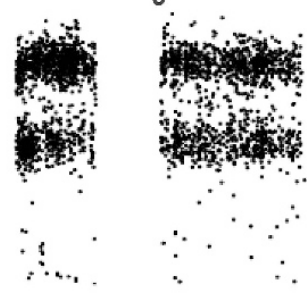

14

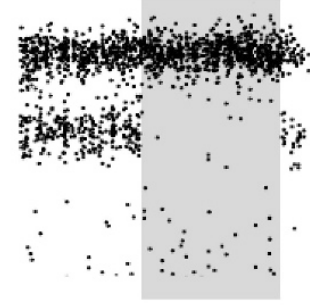

19

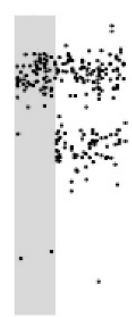

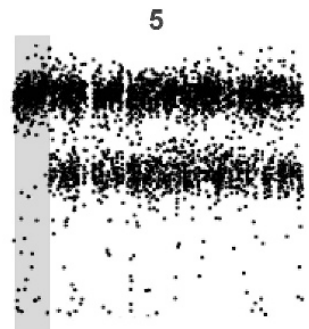

10

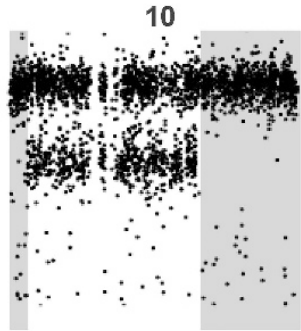

15

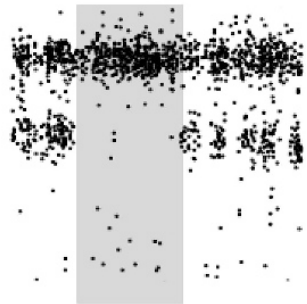

20

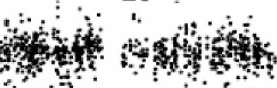

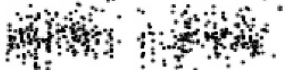

Figure 2 CMA-COMP array showing a patient with consanguinity as demonstrated by the multiple blocks of AOH (shaded) on numerous chromosomes.

500 bp. A comparison of the distribution of the probes of the CMACOMP to two commercial SNP arrays for the intragenic deletions involving exons of the VPS13B gene (Table 5, cases 6 and 7 ) is provided in Figures $3 \mathrm{c}$ and $\mathrm{d}$. In addition to the VPS13B gene, intragenic deletions of both NRXN1 and DMD were detected in multiple patients as well as two cases involving genes that predispose to cancer.

\section{DISCUSSION}

With the addition of SNP probes to our exon coverage array, we provide a comprehensive approach for the identification of clinically relevant copy number neutral changes in addition to medically actionable CNVs in a single assay.
The principles used to guide this unique array design were to: (1) empirically select the best performing probes to maximize detection and signal to noise ratio, (2) detect CNVs known to be associated with diseases and target coverage of the exons of these known disease genes, (3) maintain $30 \mathrm{~kb}$ backbone coverage, and (4) exclude most of the known LCR regions. The rationale for our array design to maximize detection of clinically relevant genomic changes has been independently validated by the observations of Haraksingh et al. ${ }^{33}$ They compared the CNVs observed in the 1000 Genomes Project with all the currently commercially available high-resolution array platforms and concluded that the sensitivity, total number, size range, and breakpoint resolution of $\mathrm{CNV}$ calls were highest for $\mathrm{CNV}$ focused arrays that did not compromise the backbone tiling 
density of the rest of the genome. They also found that probe distribution greatly affected the performance of a platform. A disadvantage for SNP-only arrays is that the probe distribution is restricted by the non-uniform availability of informative SNPs throughout the genome. Additionally, probes that are optimized will provide maximum information for specific loci.

In this study, the CMA-COMP array, with only 60000 SNP probes, was concordant with a high-density SNP array in detecting $\mathrm{AOH}$ events and is thus an excellent tool for genome-wide screening for $\mathrm{AOH}$ in order to identify UPD, consanguinity, and map genomic intervals containing potential recessive loci. The percent of the genome manifesting $\mathrm{AOH}$ for each case was calculated for both platforms (data not shown) and there was complete concordance between the two platforms within 1-2\%. Because of the lower density of SNP probes in the CMA-COMP array, there may be an overestimation of the genomic size of an $\mathrm{AOH}$ event, which occurred in two cases in Table 1. In one of these cases, the Illumina array revealed a smaller $\mathrm{AOH}(6 \mathrm{Mb})$ event, and in the other case, a series of smaller discrete $\mathrm{AOH}$ events interrupted by small regions of heterodisomy were seen in the Illumina array. The CMA-COMP did not resolve the discrete interval events; instead it showed a contiguous AOH event for this region. As both of these cases involved a non-imprinted chromosome, further studies were not recommended.

Added value to the genomic analysis provided by SNP probes is the detection of uniparental isodisomy without requiring analysis of parental samples, as it is with other conventional assays that utilize STR markers. In addition, UPD of virtually any chromosome can be detected in one assay. While SNP arrays will detect virtually $100 \%$ of isodisomies, ${ }^{24}$ not all cases of uniparental heterodisomy may be detected by SNP arrays unless a heterodisomic chromosome underwent a recombination event during meiosis, resulting in

Table 3 Extent of $\mathrm{AOH}$ regions detected in patients from known consanguineous families

\begin{tabular}{|c|c|c|c|c|c|}
\hline & & CMA-COMP array & & & \\
\hline Patients & $\begin{array}{c}\text { AOH level } \\
(M b)\end{array}$ & $\begin{array}{l}\text { Number of chro- } \\
\text { mosomes involved }\end{array}$ & $\begin{array}{l}\text { Number } \\
\text { of blocks }\end{array}$ & $\begin{array}{l}\text { Parental } \\
\text { relationship }\end{array}$ & $\begin{array}{l}\text { Expected } \\
\mathrm{AOH}\end{array}$ \\
\hline Case $1^{a}$ & 707 & 16 & 23 & $\begin{array}{l}\text { First-degree } \\
\text { relatives }\end{array}$ & $\begin{array}{l}716 \mathrm{Mb} \\
(F=1 / 4)\end{array}$ \\
\hline Case 2 & 225 & 9 & 10 & First cousins & $\begin{array}{c}179 \mathrm{Mb} \\
(F=1 / 16)\end{array}$ \\
\hline Case 3 & 89 & 4 & 4 & $\begin{array}{l}\text { First cousins } \\
\text { once removed }\end{array}$ & $\begin{array}{c}90 \mathrm{Mb} \\
(F=1 / 32)\end{array}$ \\
\hline Case 4 & 82 & 3 & 3 & $\begin{array}{l}\text { First cousins } \\
\text { once removed }\end{array}$ & $\begin{array}{c}90 \mathrm{Mb} \\
(F=1 / 32)\end{array}$ \\
\hline Case 5 & 58 & 3 & 3 & $\begin{array}{l}\text { Second cousins } \\
\text { once removed }\end{array}$ & $\begin{array}{c}22.5 \mathrm{Mb} \\
(F=1 / 128)\end{array}$ \\
\hline
\end{tabular}

a Same as case 3 in Table 2. isodisomy interrupted by regions of heterozygosity, reflecting the presence of heterodisomy (hetero-isoUPD). In such cases, heterodisomy appears as a large block of $\mathrm{AOH}$ (isodisomic segment) confined to a single chromosome. Recently, Papenhausen et $a l^{22}$ found that their cohort of nine confirmed UPD cases all showed an $\mathrm{AOH}$ block within the affected chromosome. Similar findings were also observed in five confirmed UPD cases and an additional three were consistent with the phenotype by Bruno et al. ${ }^{34}$ However, not all $\mathrm{AOH}$ regions confined to a single chromosome indicate the presence of heterodisomy, as shown in our patients with $\mathrm{AOH}$ on chromosome 14. Similar observations were reported by Papenhausen et al, ${ }^{22}$ who showed that only 29 out of 46 cases with a single segmental $\mathrm{AOH}$ event were confirmed to be UPD. Therefore, a single region of $\mathrm{AOH}$ could be a random finding representing a region of identity by descent or linkage disequilibrium (LD) because of a low recombination rate.

Detection of isodisomy has obvious clinical relevance for cases in which chromosomes bearing imprinted genomic regions are involved, but it is also relevant in the context of autosomal recessive (AR) disorders. Most of the non-imprinted UPD cases described so far were ascertained by detection of a homozygous mutation in autosomal recessive genes that did not follow the expected Mendelian allele transmission pattern. ${ }^{26}$ From a genetic counseling perspective, detection of isodisomy or hetero-isoUPD modifies recurrence risk for AR disease from $25 \%$ to $<1 \%$. The importance of parental follow-up studies to confirm carrier status in cases in which a homozygous mutation was detected in the child cannot be underemphasized.

The identification of $\mathrm{AOH}$ has been previously employed for the determination of the genetic defects underlying genetically heterogeneous recessive disorders by a homozygosity mapping approach in a research setting. $\mathrm{AOH}$ regions determined by genome-wide SNPs analysis allows delineation of the critical disease-associated genomic interval and the number of potential candidate genes. Analysis of SNP data obtained for both affected and unaffected individuals enables further refinement of $\mathrm{AOH}$ regions and subsequent identification of candidate genes. Implementation of SNP arrays in clinical diagnostics allows the clinician to take advantage of this approach in their daily practice, especially because these data could be combined with clinically available whole-exome sequencing approaches. Clinicians can also utilize SNP genotyping information to devise a cost-effective strategy for determining the molecular etiology of genetically heterogeneous autosomal recessive diseases (e.g., deafness, retinitis pigmentosa, etc) by targeting sequence analysis for a suspected disease gene if it is located in an $\mathrm{AOH}$ block. Such was the case for the patients identified with SCID and Usher syndrome (Table 4).

The CMA-COMP array also has the advantage of detecting intragenic copy number changes. While intragenic deletions and duplications are known to account for a significant portion of diseasecausing mutations for genes such as $D M D$, only more recently with the implementation of array CGH are intragenic deletions and duplications being detected for many other genes. Examples of

Table 4 Correlation of $\mathrm{AOH}$ regions with autosomal recessive disorders

\begin{tabular}{|c|c|c|c|c|c|}
\hline AR disorder & Genes related to diagnosis & Total $\mathrm{AOH}>10 \mathrm{Mb}$ & Gene within $\mathrm{AOH}$ block & $A O H$ size $(M b)$ & Comment \\
\hline Cohen syndrome & $V S P 13 B$ & 29 & $V S P 13 B$ & 11 & Homozygous exonic deletion detected \\
\hline HHH syndrome & $S L C 25 A 15$ & 270 & $S L C 25 A 15$ & 40 & Homozygous mutation in SLC25A15 detected \\
\hline SCID & 18 genes & 332 & $C D 3 D$ & 8 & Homozygous mutation in $C D 3 D$ detected \\
\hline Usher syndrome & Dozens of genes & 214 & USH2A & 18 & \\
\hline
\end{tabular}


a
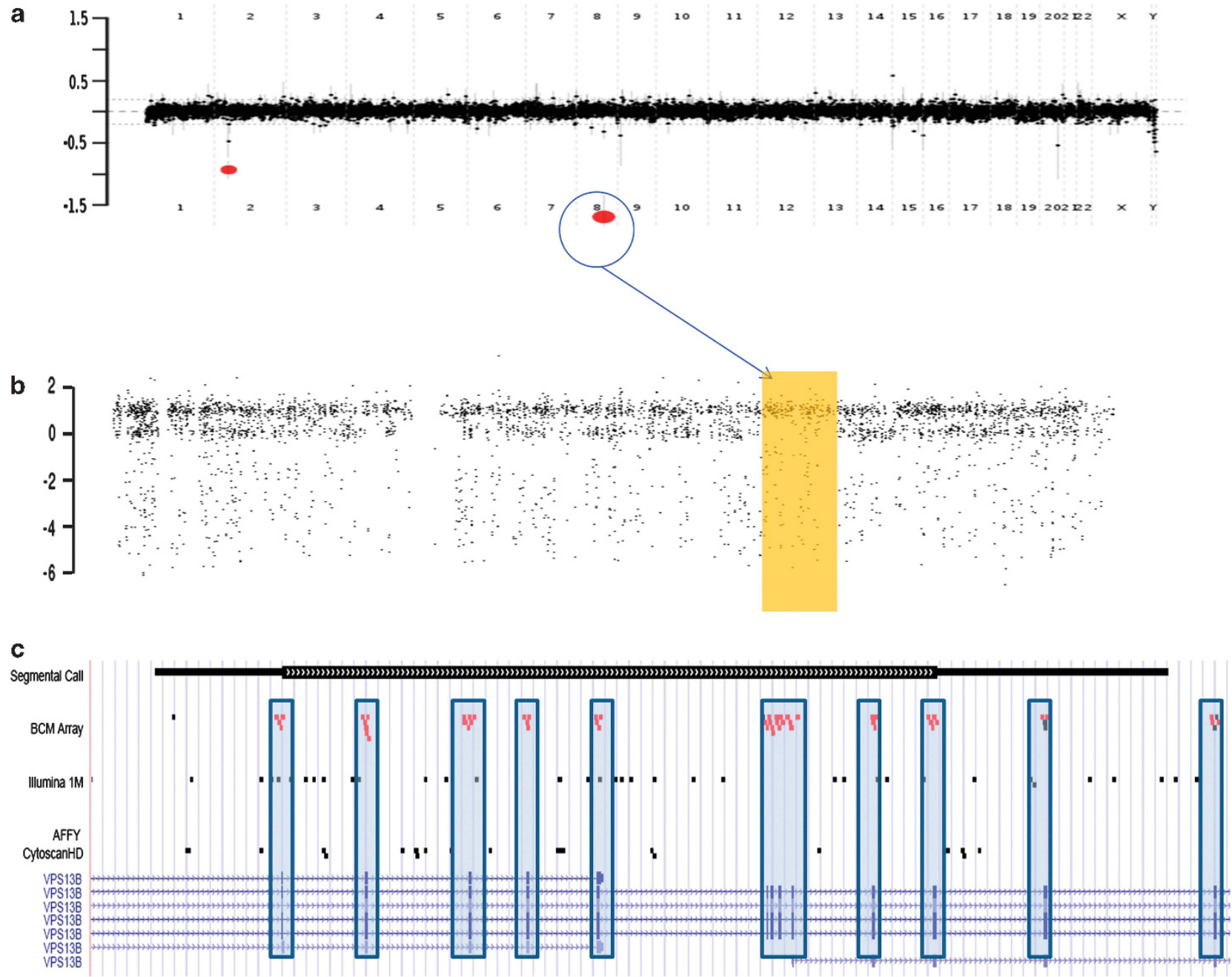

d

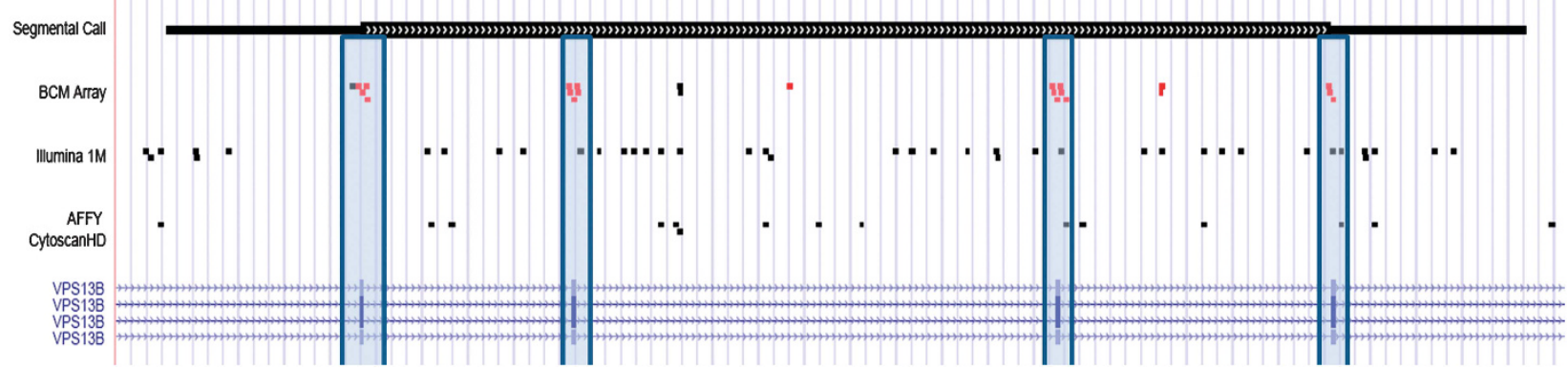

Figure 3 (a) A 52-kb homozygous deletion involving exons 4-14 of the VSP13B gene in a patient with Cohen syndrome detected by CMA-COMP array. (b) shows the SNP data plot with an AOH region on chromosome 8, wherein the VSP13B gene is located. (c) and (d) Comparison of the probe distribution between the exon-targeted CMA-COMP array and two other commercial SNP arrays for the portion of the VSP13B gene deleted in cases 6 and 7 (Table 4 ) (c) Case 6 (same case as in (a)) with a deletion of exons 4-14 (red dots represent the deleted oligos in the exons). Note that the locations of the SNPs (black dots) are outside of almost all of the exons, and therefore single exon deletions would not be detectable by these SNP arrays. (d) Case 7 in Table 4 - red dots represent the deleted oligonucleotides corresponding to exons 22-25 (represented by the hatch marks at the bottom of the figure) as detected by the CMA-COMP array. The black dots represent the SNP distribution within the same region. Again, note that most SNP probes are located within introns.

haploinsufficient, intragenic deletions and the molecular mechanism and possible consequences of these intragenic CNVs have been previously reported. ${ }^{4,12,13,16,35,36}$ Detection of intragenic copy number changes also has a role in the diagnosis of autosomal recessive disease, as demonstrated by the case with an intragenic heterozygous deletion of the VPS13B gene (case 7 in Table 4), which is associated with Cohen syndrome. Consistently, deletions have been reported as an important cause of Cohen syndrome. ${ }^{37}$

Interestingly, genotype-phenotype correlations are being described for deletions occurring at different locations within a gene. For 
Table 5 Pathogenic exonic CNVs detected by CMA-COMP array

\begin{tabular}{|c|c|c|c|c|c|c|c|}
\hline Case & Gene & Disease association & Exon(s) & $\begin{array}{l}\text { Copy number } \\
\text { change }\end{array}$ & $\begin{array}{c}\text { Chromosome } \\
\text { Location }\end{array}$ & Inheritance & Gender \\
\hline 1 & $N R X N 1$ & Autism and developmental disorders & 23 & Loss & $2 \mathrm{p} 16.3$ & Unknown & $\mathrm{F}$ \\
\hline 3 & NRXN1 & Autism and developmental disorders & $4-5$ & Loss & $2 \mathrm{p} 16.3$ & Unknown & M \\
\hline 4 & MSH6 & Hereditary nonpolyposis colorectal cancer & $5-6$ & Loss & $2 \mathrm{p} 16.3$ & Unknown & $\mathrm{F}$ \\
\hline 5 & TGFB1 & Corneal dystrophy & $8-10$ & Loss & $5 q 31.1$ & Unknown & $\mathrm{F}$ \\
\hline 8 & BRCA2 & Increased risk of cancer & $13-14$ & Loss & $13 q 13.1$ & Unknown & M \\
\hline 9 & UBE3A & Angelman syndrome & $7-14$ & Loss & $15 q 11.2$ & Maternal & $\mathrm{F}$ \\
\hline 10 & CHRNA7 & Intellectual disability and seizures & Entire gene & Loss & $15 q 13.3$ & Unknown & $\mathrm{F}$ \\
\hline 11 & ANKRD11 & KBG syndrome & $4-13$ & Loss & $16 q 24.3$ & Unknown & $\mathrm{F}$ \\
\hline 12 & ANKRD11 & KBG syndrome & $2-3$ & Loss & $16 q 24.3$ & Unknown & $\mathrm{F}$ \\
\hline 16 & $D M D$ & Duchenne muscular dystrophy & $63-64$ & Gain & Xp21.1 & Unknown & M \\
\hline 17 & $D M D$ & Duchenne muscular dystrophy & $49-51$ & Loss & Xp21.1 & Maternal & M \\
\hline 18 & MED12 & FG syndrome and Lujan-Fryns syndrome & $25-28$ & Gain & Xq13.1 & De novo & M \\
\hline 19 & GPC3 & Simpson-Golabi-Behmel syndrome & 7 & Gain & $\mathrm{Xq} 26.2$ & Unknown & M \\
\hline 20 & FMR1 & X-linked intellectual disability & Entire gene & Gain & $\mathrm{Xq} 27.3$ & Unknown & M \\
\hline 21 & LICAM & X-linked hydrocephalus & $1-2$ & Loss & Xq28 & Unknown & M \\
\hline
\end{tabular}

example, the more C-terminal deletions, such as case 1 (Table 5), including those affecting the beta isoform of Neurexin-1, present with an increased head size and a high frequency of epilepsy (88\%) when compared with more N-terminal deletions of NRXN1, as seen in cases 2 and 3 (Table 5). Therefore, increasingly specific genotypephenotype predictions are becoming available to assist with the genetic counseling of the copy number changes identified by array studies. ${ }^{28,35}$

However, genetic counseling can also become complicated by incidental findings, such as the intragenic deletions within MSH6 in case 4 and BRCA2 in case 8 (Table 5), which confers an increased lifetime susceptibility to cancer in a 13-year-old female and a 10-year-old male, respectively. These findings require careful counseling to address prognosis and surveillance. These findings also have obvious implications for other family members that may also have inherited the CNV. While unexpected, these findings can have a profound impact on optimizing medical management and prevention for the whole family. As intragenic deletions of these genes have been observed, ${ }^{36,38}$ it is expected that incidental findings such as these will occur as arrays with targeted exonic coverage are increasingly used in clinical practice.

In conclusion, combining both array CGH and SNP genotyping in a single platform optimizes the clinical diagnostic capability by offering the simultaneous detection of copy number neutral changes and small intragenic copy number changes.

\section{CONFLICT OF INTEREST}

Drs Wiszniewska, Bi, Shaw, Stankiewicz, Lalani, Probst, Scaglia, Beaudet, Lupski, Eng, Cheung, Bacino, Patel are the faculty of the Department of Molecular and Human Genetics at Baylor College of Medicine, which derives revenue from molecular genetic testing offered in the Medical Genetics Laboratory. Dr Lupski is a paid consultant for Athena Diagnostics, holds stock ownership in 23andMe, Inc. and Ion Torrent Systems, Inc., and is a co-inventor on multiple United States and European patents related to molecular diagnostics. The remaining authors declare no conflict of interest.

1 Lupski JR, Belmont JW, Boerwinkle E, Gibbs RA: Clan genomics and the complex architecture of human disease. Cell 2011; 147: 32-43.

2 Conrad DF, Pinto D, Redon $\mathrm{R}$ et al: Origins and functional impact of copy number variation in the human genome. Nature 2010; 464: 704-712.

3 lafrate AJ, Feuk L, Rivera MN et al: Detection of large-scale variation in the human genome. Nat Genet 2004; 36: 949-951.

4 Mills RE, Walter K, Stewart C et al: Mapping copy number variation by population-scale genome sequencing. Nature 2011; 470: 59-65.

5 Redon R, Ishikawa S, Fitch KR et al: Global variation in copy number in the human genome. Nature 2006; 444: 444-454.

6 Sebat J, Lakshmi B, Troge J et al: Large-scale copy number polymorphism in the human genome. Science 2004; 305: 525-528.

7 Tuzun E, Sharp AJ, Bailey JA et al: Fine-scale structural variation of the human genome. Nat Genet 2005; 37: 727-732.

8 Boone PM, Liu P, Zhang F et al: Alu-specific microhomology-mediated deletion of the final exon of SPAST in three unrelated subjects with hereditary spastic paraplegia. Genet Med 2011; 13: 582-592.

9 Beaudet AL, Belmont JW: Array-based DNA diagnostics: let the revolution begin. Annu Rev Med 2008; 59: 113-129.

10 Lee C, lafrate AJ, Brothman AR: Copy number variations and clinical cytogenetic diagnosis of constitutional disorders. Nat Genet 2007; 39(7 Suppl): S48-S54.

11 McCarroll SA: Copy number variation and human genome maps. Nat Genet 2010; 42 365-366.

12 Pinto D, Pagnamenta AT, Klei L et al: Functional impact of global rare copy number variation in autism spectrum disorders. Nature 2010; 466: 368-372.

13 Stankiewicz P, Beaudet AL: Use of array CGH in the evaluation of dysmorphology, malformations, developmental delay, and idiopathic mental retardation. Curr Opin Genet Dev 2007; 17: 182-192.

14 Lupski JR: Brain copy number variants and neuropsychiatric traits. Biol Psychiatr 2012; 72: 617-619.

15 Miller DT, Adam MP, Aradhya S et al: Consensus statement: chromosomal microarray is a first-tier clinical diagnostic test for individuals with developmental disabilities or congenital anomalies. Am J Hum Genet 2010; 86: 749-764.

16 Boone PM, Bacino CA, Shaw CA et al: Detection of clinically relevant exonic copynumber changes by array CGH. Hum Mutat 2010; 31: 1326-1342.

17 Tsai AC, Dossett CJ, Walton CS et al: Exon deletions of the EP300 and CREBBP genes in two children with Rubinstein-Taybi syndrome detected by aCGH. Eur J Hum Genet $2011 ; 19: 43-49$ 
18 Conlin LK, Thiel BD, Bonnemann CG et al: Mechanisms of mosaicism, chimerism and uniparental disomy identified by single nucleotide polymorphism array analysis. Hum Mol Genet 2010; 19: 1263-1275.

19 de Leeuw N, Hehir-Kwa JY, Simons A et al: SNP array analysis in constitutional and cancer genome diagnostics - copy number variants, genotyping and quality control. Cytogenet Genome Res 2011; 135: 212-221.

20 McQuillan R, Leutenegger AL, Abdel-Rahman R et al: Runs of homozygosity in European populations. Am J Hum Genet 2008; 83: 359-372.

21 Altug-Teber O, Dufke A, Poths $\mathrm{S}$ et al: A rapid microarray based whole genome analysis for detection of uniparental disomy. Hum Mutat 2005; 26: 153-159.

22 Papenhausen $\mathrm{P}$, Schwartz S, Risheg $\mathrm{H}$ et al: UPD detection using homozygosity profiling with a SNP genotyping microarray. Am J Med Genet A 2011; 155A: 757-768.

23 Engel E: A new genetic concept: uniparental disomy and its potential effect, isodisomy. Am J Med Genet 1980; 6: 137-143.

24 Spence JE, Perciaccante RG, Greig GM et al: Uniparental disomy as a mechanism for human genetic disease. Am J Hum Genet 1988; 42: 217-226.

25 Robinson WP, Christian SL, Kuchinka BD et al: Somatic segregation errors predominantly contribute to the gain or loss of a paternal chromosome leading to uniparental disomy for chromosome 15. Clin Genet 2000; 57: 349-358.

26 Kotzot D: Complex and segmental uniparental disomy updated. J Med Genet 2008; 45: 545-556.

27 Ou Z, Kang SH, Shaw CA et al: Bacterial artificial chromosome-emulation oligonucleotide arrays for targeted clinical array-comparative genomic hybridization analyses. Genet Med 2008; 10: 278-289.

28 Boone PM, Soens ZT, Campbell IM et al: Incidental copy-number variants identified by routine genome testing in a clinical population. Genet Med 2012; 15: 45-54.

29 Kearney HM, Thorland EC, Brown KK, Quintero-Rivera F, South ST: American College of Medical Genetics standards and guidelines for interpretation and reporting of postnatal constitutional copy number variants. Genet Med 2011; 13: 680-685.
30 de Leeuw N, Dijkhuizen T, Hehir-Kwa JY et al: Diagnostic interpretation of array data using public databases and internet sources. Hum Mutat 2012; 33: 930-940.

31 Abu-Amero SN, Ali Z, Abu-Amero KK, Stanier P, Moore GE: An analysis of common isodisomic regions in five mUPD 16 probands. J Med Genet 1999; 36: 204-207.

32 Burrage LC, Person RE, Flores A et al: De novo interstitial duplication of 15q11.2-q13.1 with complex maternal uniparental trisomy for the 15q11-q13 region in a patient with Prader-Willi syndrome. Am J Med Genet A 2012; 158A: 2557-2563.

33 Haraksingh RR, Abyzov A, Gerstein M, Urban AE, Snyder M: Genome-wide mapping of copy number variation in humans: comparative analysis of high resolution array platforms. PLoS One 2011; 6: e27859.

34 Bruno DL, White SM, Ganesamoorthy D et al: Pathogenic aberrations revealed exclusively by single nucleotide polymorphism (SNP) genotyping data in 5000 samples tested by molecular karyotyping. J Med Genet 2011; 48: 831-839.

35 Schaaf CP, Boone PM, Sampath S et al: Phenotypic spectrum and genotype-phenotype correlations of NRXN1 exon deletions. Eur J Hum Genet 2012; 20: 1240-1247.

36 van der Klift H, Wijnen J, Wagner A et al: Molecular characterization of the spectrum of genomic deletions in the mismatch repair genes MSH2, MLH1, MSH6, and PMS2 responsible for hereditary nonpolyposis colorectal cancer (HNPCC). Genes Chromosomes Cancer 2005; 44: 123-138.

37 Balikova I, Lehesjoki AE, de Ravel TJ et al: Deletions in the VPS13B (COH1) gene as a cause of Cohen syndrome. Hum Mutat 2009; 30: E845-E854.

38 Judkins T, Rosenthal E, Arnell $\mathrm{C}$ et al: Clinical significance of large rearrangements in BRCA1 and BRCA2. Cancer 2012; 118: 5210-5216.

(c) (i) (-) $\Theta$ This work is licensed under a Creative Commons Attribution-NonCommercial-NoDerivs 3.0 Unported License. To view a copy of this license, visit http://creativecommons. org/licenses/by-nc-nd/3.0/

Supplementary Information accompanies this paper on European Journal of Human Genetics website (http://www.nature.com/ejhg) 